Document downloaded from:

http://hdl.handle.net/10251/108051

This paper must be cited as:

Motos Izquierdo, J.; Planells Gilabert, MJ.; Talavera Usano, CF. (2013). A Note on Variable Exponent Hörmander Spaces. Mediterranean Journal of Mathematics. 10(3):1419-1434. doi:10.1007/s00009-013-0268-y

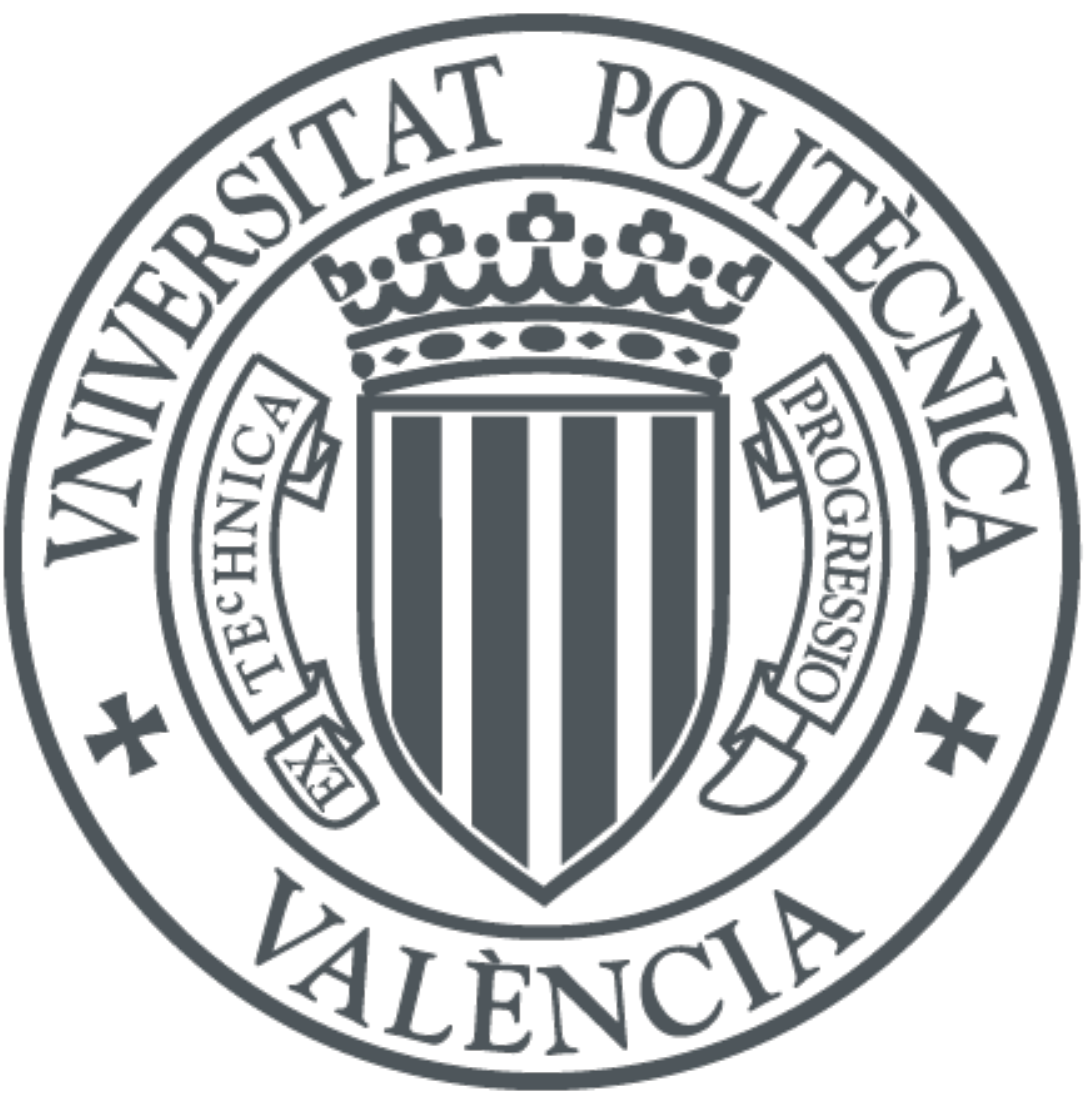

The final publication is available at

https://doi.org/10.1007/s00009-013-0268-y

Copyright Springer-Verlag

Additional Information 


\title{
A note on variable exponent Hörmander spaces
}

\author{
Joaquín Motos, María Jesús Planells and César Felix Talavera
}

\begin{abstract}
In this paper we introduce the variable exponent Hörmander spaces

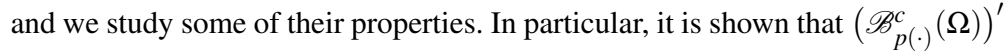
is isomorphic to $\mathscr{B} \frac{\text { loc }}{p^{\prime}(\cdot)}(\Omega)\left(\Omega\right.$ open set in $\mathbb{R}^{n}, p_{-}>1$ and the Hardy-Littlewood maximal operator $M$ is bounded in $\left.L_{p(\cdot)}\right)$ extending a Hörmander's result to our context. As a consequence, a number of results on sequence space representations of variable exponent Hörmander spaces are given.
\end{abstract}

Mathematics Subject Classification (2010). 46F05; 46E10; 46E16; 46E13.

Keywords. Variable exponent Lebesgue spaces, variable exponent Lebesgue spaces of entire analytic functions, Hardy-Littlewood maximal operator, Hörmander spaces.

\section{Introduction and notation}

\subsection{Introduction}

The Lebesgue spaces $L_{p(\cdot)}$ with variable exponent and the corresponding Sobolev spaces $W_{p(\cdot)}^{m}$ have been intensively investigated during the last years (see the recent book of Diening et al. [3]). These spaces are of interest in their own right and also have applications to PDE of non-standard growth (see e.g. [3, Chapter 13]) and to modelling electrorheological fluids and to image restoration (see [3, Chapter 14]). Our paper lies in this field of variable exponent function spaces. We introduce the variable exponent Hörmander spaces $\mathscr{B}_{p(\cdot)}, \mathscr{B}_{p(\cdot)}^{c}(\Omega)$ and $\mathscr{B}_{p(\cdot)}^{\text {loc }}(\Omega)$ (it is well known that the Hörmander spaces $\mathscr{B}_{p, k}, \mathscr{B}_{p, k}^{c}(\Omega)$ and $\mathscr{B}_{p, k}^{\text {loc }}(\Omega)$ play a crucial role in the theory of linear partial differential operators (see e.g. [8], [24], [6], [15], [16], [17])) and we study some of their properties. We also give a number of results on sequence space representations of the introduced spaces.

The organization of the paper is as follows. Section 2 contains some basic facts about variable exponent Lebesgue spaces and the definition of variable exponent Lebesgue spaces of entire analytic functions. In Section 3 we introduce the variable exponent Hörmander spaces $\mathscr{B}_{p(\cdot)}, \mathscr{B}_{p(\cdot)}^{c}(\Omega)$ and $\mathscr{B}_{p(\cdot)}^{\text {loc }}(\Omega)$ and we study some of their properties when the Hardy-Littlewood maximal operator $M$ is bounded 
in $L_{p(\cdot) / p_{0}}$ for some $0<p_{0}<p_{-}$(convolution, density, completeness, embedding theorems, multiplication operators) and, by using Fourier multipliers and a result of Diening [2], we obtain a sequence space representation of the space $\mathscr{B}_{p(\cdot)}^{c}(] a, b[)$ (see Theorem 3.5/5). We also extend a result of Hörmander [8, Chapter XV, 15.2] to the variable exponent Hörmander spaces $\mathscr{B}_{p(\cdot)}^{c}(\Omega)$ (see Remark 3.6/2). In Section 4 we show that $\left(\mathscr{B}_{p(\cdot)}^{c}(\Omega)\right)^{\prime}$ is isomorphic to $\mathscr{B}_{p^{\prime}(\cdot)}^{\text {loc }}(\Omega)$ (see also [8, Chapter $\mathrm{XV}]$ and [16]) and another result on sequence space representation is given. Finally, two questions on complex interpolation and on sequence space representation of variable exponent Hörmander spaces are proposed.

\subsection{Notation}

Let $E$ and $F$ be topological linear spaces over $\mathbb{C}$. If $E$ and $F$ are (topologically) isomorphic we put $E \simeq F$. The (topological) dual of $E$ is denoted by $E^{\prime}$ and is given the strong topology (i.e. the topology of uniform convergence on all the bounded subsets of $E$ ). We put $E \hookrightarrow F$ if $E$ is a linear subspace of $F$ and the canonical injection is continuous (if $E$ is also dense in $F$ we replace $\hookrightarrow$ by $\stackrel{d}{\hookrightarrow}$ ). If $\left\{E_{n}\right\}_{n=1}^{\infty}$ is a sequence of Banach spaces, $\prod_{n=1}^{\infty} E_{n}$ denotes the topological product of the spaces $E_{n}$ and $\oplus_{n=1}^{\infty} E_{n}$ their locally convex direct sum. The Fréchet space defined by the projective sequence of Banach spaces $E_{n}$ and linking maps $A_{n}$ will be denoted by $\operatorname{proj}_{n}\left(E_{n}, A_{n}\right)$ (or $\operatorname{proj}_{n} E_{n}$, for short). If $\left\{E_{n}\right\}_{n=1}^{\infty}$ is a sequence of topological linear spaces such that $E_{n} \hookrightarrow E_{n+1}$ for each $n$, then their inductive limit is denoted by $\operatorname{ind}_{n} E_{n}$ (see [9], [10]).

If $f \in L_{1}\left(\mathbb{R}^{n}\right)$ the Fourier transform of $f, \hat{f}$ or $\mathscr{F} f$, is defined by $\hat{f}(\xi)=$ $\int_{\mathbb{R}^{n}} f(x) e^{-i \xi x} d x$. If $f$ is a function on $\mathbb{R}^{n}$, then $\tilde{f}(x)=f(-x)$ for $x \in \mathbb{R}^{n} . B_{r}$ is the closed Euclidean ball $\{x:|x| \leq r\}$ in $\mathbb{R}^{n} . C_{0}^{\infty}\left(\mathbb{R}^{n}\right)\left(=\mathscr{D}\left(\mathbb{R}^{n}\right)\right), C_{0}^{\infty}(\Omega)(=\mathscr{D}(\Omega))$ and $S\left(\mathbb{R}^{n}\right)$ are the usual Schwartz spaces (in the last space the norms $\max _{|\alpha| \leq m} \sup _{x \in \mathbb{R}^{n}}$ $\left(1+|x|^{2}\right)^{m}\left|\partial^{\alpha} \varphi(x)\right|, m=0,1,2, \ldots$, are denoted by $\left.|\varphi|_{m}\right) . \mathscr{D}^{\prime}\left(\mathbb{R}^{n}\right), \mathscr{D}^{\prime}(\Omega)$ and $S^{\prime}\left(\mathbb{R}^{n}\right)$ are their corresponding duals. $\mathscr{E}^{\prime}(K)\left(K\right.$ compact in $\left.\mathbb{R}^{n}\right)$ is the set of distributions on $\mathbb{R}^{n}$ with supports contained in $K$. $O_{M}$ is the space of all functions $\varphi \in C^{\infty}\left(\mathbb{R}^{n}\right)$ such that $\varphi \psi \in S\left(\mathbb{R}^{n}\right)$ for all $\psi \in S\left(\mathbb{R}^{n}\right)$. The Fourier transform in $S^{\prime}\left(\mathbb{R}^{n}\right)$ is also denoted by ^ (or $\left.\mathscr{F}\right)$. If $u \in S^{\prime}\left(\mathbb{R}^{n}\right)$, $\tilde{u}$ is defined by $\langle\varphi, \tilde{u}\rangle=\langle\tilde{\varphi}, u\rangle$ for all $\varphi \in S\left(\mathbb{R}^{n}\right)$; thus $\sim$ coincides with the operator $(2 \pi)^{-n} \mathscr{F}^{2}$. In general, we will consider function spaces defined on the whole Euclidean space $\mathbb{R}^{n}$. So, in what follows, we shall omit the " $\mathbb{R}^{n}$ " of their notation. The letter $C$ will always denote a positive constant, not necessarily the same at each ocurrence.

\section{Preliminaries}

In this section we collect some basic facts about variable exponent spaces and we give the definition of variable Lebesgue spaces of entire analytic functions.

\subsection{Variable exponent spaces}

If $p(\cdot)$ is a measurable function on $\mathbb{R}^{n}$ with range in $[1, \infty]$, we put $\mathbb{R}_{\infty}^{n}=\left\{x \in \mathbb{R}^{n}\right.$ : $p(x)=\infty\}, p_{-}=\operatorname{ess~inf}_{x \in \mathbb{R}^{n}} p(x), p_{+}=\operatorname{ess~sup}_{x \in \mathbb{R}^{n}} p(x)$, and we define the modular 
functional

$$
\rho(f):=\int_{\mathbb{R}^{n} \backslash \mathbb{R}_{\infty}^{n}}|f(x)|^{p(x)} d x+\|f\|_{L_{\infty}\left(\mathbb{R}_{\infty}^{n}\right)} .
$$

$L_{p(\cdot)}$ denotes the set of all complex-valued measurable functions on $\mathbb{R}^{n}$ such that for some $\lambda>0, \rho(f / \lambda)<\infty$. This set becomes a Banach space when equipped with the Luxemburg norm

$$
\|f\|_{p(\cdot)}:=\inf \{\lambda>0: \rho(f / \lambda) \leq 1\} .
$$

These spaces are referred to as variable exponent Lebesgue spaces since they generalize the standard Lebesgue spaces. In this paper, we shall also consider analogous spaces to classical Lebesgue spaces $L_{p}, 0<p<1$, with variable exponents. Define $\mathscr{P}^{0}$ to be the set of all measurable functions on $\mathbb{R}^{n}$ with range in $(0, \infty)$ such

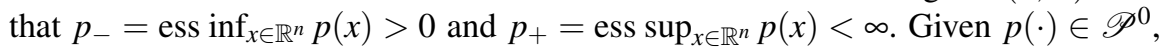
we also define the space $L_{p(\cdot)}$ as above. This is equivalent to defining it to be the set of all measurable functions $f$ such that $|f|^{p_{0}} \in L_{q(\cdot)}$, where $0<p_{0} \leq p_{-}$and $q(x)=\frac{p(x)}{p_{0}}$. We can define a quasi-norm on this space by

$$
\|f\|_{p(\cdot)}:=\left\||f|^{p_{0}}\right\|_{q(\cdot)}^{1 / p_{0}}\left(=\inf \left\{\lambda>0: \int_{\mathbb{R}^{n}}\left(\frac{|f(x)|}{\lambda}\right)^{p(x)} d x \leq 1\right\}\right)
$$

(see [1]). With this quasi-norm $L_{p(\cdot)}$ becomes a quasi-Banach space.

Next we give some basic results about variable Lebesgue spaces. Their proofs can be found in [3] (see also [11]).

Lemma 2.1. If $p(\cdot) \in \mathscr{P}^{0}$, then $\left\|f_{k}-f\right\|_{p(\cdot)} \rightarrow 0$ if and only if $\int_{\mathbb{R}^{n}}\left|f_{k}(x)-f(x)\right|^{p(x)} d x$ $\rightarrow 0$. If $\left\|f_{k}-f\right\|_{p(\cdot)} \rightarrow 0$ then there exists a subsequence of $\left(f_{k}\right)$ which converges a.e. to $f$.

Lemma 2.2 (generalized Hölder inequality). Let $p(\cdot): \mathbb{R}^{n} \rightarrow[1, \infty]$. If $f \in L_{p(\cdot)}$ and $g \in L_{p^{\prime}(\cdot)}$, then

$$
\int_{\mathbb{R}^{n}}|f(x) g(x)| d x \leq C\|f\|_{p(\cdot)}\|g\|_{p^{\prime}(\cdot)}
$$

where $p^{\prime}(\cdot)$ is the conjugate exponent function defined by $\frac{1}{p(x)}+\frac{1}{p^{\prime}(x)}=1, x \in \mathbb{R}^{n}$.

Lemma 2.3. Let $p(\cdot): \mathbb{R}^{n} \rightarrow[1, \infty]$. The natural mapping

$$
I: L_{p^{\prime}(\cdot)} \rightarrow\left(L_{p(\cdot)}\right)^{\prime}: g \rightarrow\langle f, I(g)\rangle=\int_{\mathbb{R}^{n}} f(x) g(x) d x
$$

is an isomorphism if and only if $p_{+}<\infty$. The space $L_{p(\cdot)}$ is reflexive if and only if $1<p_{-} \leq p_{+}<\infty$.

\section{Lemma 2.4.}

1. Let $p(\cdot): \mathbb{R}^{n} \rightarrow[1, \infty]$. Then $S \hookrightarrow L_{p(\cdot)} \hookrightarrow L_{1}^{\text {loc }}, S^{\prime}$. Furthermore, if $p_{+}<\infty, C_{0}^{\infty}$ and $S$ are dense in $L_{p(\cdot)}$ and the mapping $S \times L_{p(\cdot)} \rightarrow L_{p(\cdot)}:(\varphi, f) \rightarrow \varphi f$ is continuous.

2. If $p(\cdot) \in \mathscr{P}^{0}, S \hookrightarrow L_{p(\cdot)}$ and the mapping $S \times L_{p(\cdot)} \rightarrow L_{p(\cdot)}:(\varphi, f) \rightarrow \varphi f$ is continuous. 
Many classical operators in harmonic analysis (maximal operators, CalderónZygmund operators, fractional integrals, ...) are bounded in $L_{p(\cdot)}$ whenever the Hardy-Littlewood maximal operator $M$ is bounded in $L_{p(\cdot)}$ (c.f., e.g. [1], [2] and [3]). The next lemma provides a sufficient condition on $p(\cdot)$ for $M$ to be bounded in $L_{p(\cdot)}$ and one important consequence of the Diening's characterization of variable Lebesgue spaces on which the maximal operator $M$ is bounded:

\section{Lemma 2.5.}

1. Let $p(\cdot): \mathbb{R}^{n} \rightarrow[1, \infty]$ be such that $1<p_{-} \leq p_{+}<\infty$. Suppose that $p(\cdot)$ satisfies

$$
|p(x)-p(y)| \leq \frac{C}{-\log |x-y|}, \quad|x-y| \leq \frac{1}{2}
$$

and

$$
|p(x)-p(y)| \leq \frac{C}{\log (e+|x|)}, \quad|x| \leq|y| .
$$

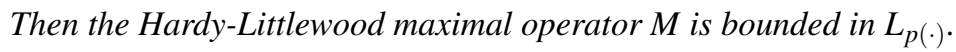

2. If $1<p_{-} \leq p_{+}<\infty$, then the following conditions are equivalent:

(a) $M$ is bounded in $L_{p(\cdot) \text {, }}$

(b) $M$ is bounded in $L_{p^{\prime}(\cdot)}$,

(c) $M$ is bounded in $L_{p(\cdot) / q}$ for some $1<q<p_{-}$,

(d) $M$ is bounded in $L_{(p(\cdot) / q)^{\prime}}$ for some $1<q<p_{-}$.

Remark 2.6. In spite of the previous results, the variable Lebesgue spaces have a number of undesired properties. For example, these spaces are not translation invariant and so the Young's inequality cannot be generalized to the spaces $L_{p(\cdot)}$ for non-constant $p(\cdot)$ (see [3] and [11]).

\subsection{Variable exponent Lebesgue spaces of entire analytic functions}

If $K$ is a compact subset of $\mathbb{R}^{n}, \mu$ is a positive Borel measure on $\mathbb{R}^{n}$ and $0<p \leq \infty$, then

$$
L_{p}^{K}(\mu):=\left\{f \in S^{\prime}: \operatorname{supp} \hat{f} \subset K, f \in L_{p}(\mu)\right\}
$$

$\left(L_{p}^{K}:=L_{p}^{K}(\mu)\right.$ if $\mu$ is the Lebesgue measure). $\left(L_{p}^{K}(\mu),\|\cdot\|_{p}\right)$ is a quasi-Banach (Banach if $p \geq 1$ ) space (see [23], [19]; see also [20]).

If $K$ is a compact subset of $\mathbb{R}^{n}$ and $p(\cdot) \in \mathscr{P}^{0}$, then

$$
L_{p(\cdot)}^{K}:=\left\{f \in S^{\prime}: \operatorname{supp} \hat{f} \subset K,\|f\|_{p(\cdot)}<\infty\right\} .
$$

$\left(L_{p(\cdot)}^{K},\|\cdot\|_{p(\cdot)}\right)$ is a quasinormed (normed if $\left.p_{-} \geq 1\right)$ linear space. From the PaleyWiener-Schwartz theorem it follows that the elements of $L_{p(\cdot)}^{K}$ are entire analytic functions of exponential type. When $p(\cdot) \equiv p$, a constant, then $L_{p(\cdot)}^{K}=L_{p}^{K}$ with equality of quasi-norms (resp. norms). We shall denote by $S^{K}$ the collection of all $f \in S$ such that supp $\hat{f} \subset K$; obviously $S^{K} \subset L_{p(\cdot)}^{K}$. The spaces $L_{p(\cdot)}^{K}$ have been introduced in [18]. 


\section{Variable exponent Hörmander spaces}

In this section we introduce the variable exponent Hörmander spaces $\mathscr{B}_{p(\cdot)}, \mathscr{B}_{p(\cdot)}^{c}(\Omega)$ and $\mathscr{B}_{p(\cdot)}^{\text {loc }}(\Omega)$ (see Definition 3.1) and we study some of their properties when the Hardy-Littlewood maximal operator $M$ is bounded in $L_{p(\cdot) / p_{0}}$ for some $0<p_{0}<p_{-}$: convolution, density, completeness, embedding theorems, multiplication operators, sequence space representations, ...

We begin with the variable exponent (and weight $k \equiv 1$ ) counterpart of $[8$, Definition 10.1.6] (see also [8, Sections 10 and 15] and [16], [17], [24]).

Definition 3.1. Let $p(\cdot) \in \mathscr{P}^{0}$ be and let $\Omega$ be an open set in $\mathbb{R}^{n}$. Then

$$
\mathscr{B}_{p(\cdot)}:=\left\{u \in S^{\prime}: \hat{u} \in L_{p(\cdot)}\right\} \text {. }
$$

If $u \in \mathscr{B}_{p(\cdot)}$ we put $\|u\|_{\mathscr{B}_{p(\cdot)}}:=\|\hat{u}\|_{p(\cdot)} \cdot\left(\mathscr{B}_{p(\cdot)},\|\cdot\|_{\mathscr{B}_{p(\cdot)}}\right)$ is a quasi-normed space isomorphic to $\left(L_{p(\cdot)} \cap S^{\prime},\|\cdot\|_{p(\cdot)}\right)$ (a Banach space isomorphic to $L_{p(\cdot)}$ if $p_{-} \geq 1$ ). Now we consider the space

$$
\mathscr{B}_{p(\cdot)}^{c}(\Omega):=\cup\left\{\mathscr{B}_{p(\cdot)} \cap \mathscr{E}^{\prime}(K): K \Subset \Omega\right\} .
$$

If every $\mathscr{B}_{p(\cdot)} \cap \mathscr{E}^{\prime}(K)$ is equipped with the topology induced by $\mathscr{B}_{p(\cdot)}$, then $\mathscr{B}_{p(\cdot)}^{c}(\Omega)$ (endowed with the corresponding inductive linear topology) becomes a strict inductive limit

$$
\mathscr{B}_{p(\cdot)}^{c}(\Omega):=\operatorname{ind}_{K}\left[\mathscr{B}_{p(\cdot)} \cap \mathscr{E}^{\prime}(K)\right]
$$

Finally,

$$
\mathscr{B}_{p(\cdot)}^{\mathrm{loc}}(\Omega):=\left\{u \in \mathscr{D}^{\prime}(\Omega): \varphi u \in \mathscr{B}_{p(\cdot)} \text { for all } \varphi \in C_{0}^{\infty}(\Omega)\right\} .
$$

The topology of this space is generated by the seminorms (semiquasi-norms when $\left.p_{-}<1\right) u \rightarrow\|u\|_{p(\cdot), \varphi}:=\|\varphi u\|_{\mathscr{B}_{p(\cdot)}}, \varphi \in C_{0}^{\infty}(\Omega)$.

\section{Remark 3.2.}

1. $\mathscr{B}_{p(\cdot)}, \mathscr{B}_{p(\cdot)}^{c}(\Omega)$ and $\mathscr{B}_{p(\cdot)}^{\text {loc }}(\Omega)$ are called variable exponent Hörmander spaces. If $p(\cdot) \equiv p$ and $p \geq 1$, these spaces coincide with the Hörmander spaces $\mathscr{B}_{p, 1}$, $\mathscr{B}_{p, 1}^{c}(\Omega)$ and $\mathscr{B}_{p, 1}^{\text {loc }}(\Omega)$ respectively (see [8]).

2. In general, the space $\mathscr{B}_{p(\cdot)} \cap \mathscr{E}^{\prime}(K)$ ( $K$ any compact in $\left.\mathbb{R}^{n}\right)$ is not a complemented subspace of $\mathscr{B}_{p(\cdot)}$. For example, if $n>1, p(\cdot) \equiv p$ (a constant), $1<p \neq 2<\infty$ and $K=B_{1}$, then $\mathscr{B}_{p, 1} \cap \mathscr{E}^{\prime}\left(B_{1}\right)$ is not a complemented subspace of $\mathscr{B}_{p, 1}$ since $\mathscr{B}_{p, 1} \cap \mathscr{E}^{\prime}\left(B_{1}\right)$ is isomorphic (via the Fourier transform) to $L_{p}^{B_{1}}$ and this space is not a complemented subspace of $L_{p}$ by the Fefferman theorem (see [4] and [14]).

The following elementary fact will be used in the next theorem: "Let $F=$ ind $_{j} F_{j}$ be the strict inductive limit of a properly increasing sequence $F_{1} \subset F_{2} \subset \ldots$ of Banach spaces. Assume that every $F_{j}$ is a complemented subspace of $F_{j+1}$ and that $G_{j}$ is a topological complement of $F_{j}$ in $F_{j+1}$. Then the mapping $F_{1} \oplus G_{1} \oplus G_{2} \oplus$ $\cdots \rightarrow F:\left(f_{1}, g_{1}, g_{2}, \ldots\right) \rightarrow f_{1}+g_{1}+g_{2}+\cdots$ is an isomorphism". We will also need the following lemmata. 
Lemma 3.3. Let $p(\cdot) \in \mathscr{P}^{0}$. Then the bilinear mapping $\Phi: S \times \mathscr{B}_{p(\cdot)} \rightarrow \mathscr{B}_{p(\cdot)}$, defined by $\Phi(\varphi, u)=\varphi * u$, is continuous. Furthermore, for every $u \in \mathscr{B}_{p(\cdot)}, \theta_{\varepsilon} * u \rightarrow$ $u$ in $\mathscr{B}_{p(\cdot)}$ when $\varepsilon \rightarrow 0+\left(\right.$ here $\theta_{\varepsilon}(x)=\frac{1}{\varepsilon^{n}} \theta\left(\frac{x}{\varepsilon}\right)$ being $\theta$ a $C_{0}^{\infty}$ function such that $\theta \geq 0$ and $\left.\int_{\mathbb{R}^{n}} \theta(x) d x=1\right)$.

Proof. If $\varphi \in S$ and $u \in \mathscr{B}_{p(\cdot)}$ then $\varphi * u \in O_{M}$ and $\widehat{\varphi * u}=\hat{\varphi} \hat{u}$. Hence and from Lemma $2.4 / 2$ if follows that $\varphi * u \in \mathscr{B}_{p(\cdot)}$. Using again Lemma 2.4/2 and the continuity of the Fourier transform, we obtain

$$
\|\varphi * u\|_{\mathscr{B}_{p(\cdot)}}=\|\widehat{\varphi * u}\|_{p(\cdot)}=\|\hat{\varphi} \hat{u}\|_{p(\cdot)} \leq C|\hat{\varphi}|_{r}\|\hat{u}\|_{p(\cdot)} \leq C|\varphi|_{m}\|u\|_{\mathscr{B}_{p(\cdot)}}
$$

(here $r, m \in \mathbb{N}_{0}$ are independent both of $\varphi$ and $u$ ). This proves the continuity of $\Phi$. By Lemma 2.1

$$
\left\|\theta_{\varepsilon} * u-u\right\|_{\mathscr{B}_{p(\cdot)}}=\left\|\left(\hat{\theta}_{\varepsilon}-1\right) \hat{u}\right\|_{p(\cdot)}
$$

goes to zero if and only if $\int_{\mathbb{R}^{n}}\left(\left|\hat{\theta}_{\varepsilon}(x)-1\right||\hat{u}(x)|\right)^{p(x)} d x \rightarrow 0$ but the latter is a consequence of the Lebesgue dominated convergence theorem in view of the estimate $\left|\hat{\theta}_{\varepsilon}(x)-1\right|^{p(x)} \leq 2^{p_{+}}$, the fact that $\hat{\theta}_{\varepsilon} \rightarrow 1$ pointwise when $\varepsilon \rightarrow 0+$, and the integrability of the function $|\hat{u}(x)|^{p(x)}$.

Lemma 3.4. Let $K$ be a compact subset of $\mathbb{R}^{n}$ and let $p(\cdot) \in \mathscr{P}^{0}$ be such that $M$ is bounded in $L_{p(\cdot) / p_{0}}$ for some $0<p_{0}<p_{-}$.

1. The convolution $S \times L_{p(\cdot)}^{K} \rightarrow L_{p(\cdot)}^{K}:(\varphi, f) \rightarrow \varphi * f$ is well defined and is bilinear and continuous.

2. (Inequalities of Plancherel-Polya-Nikol'skij type) Let $\alpha$ be a multiindex. Then there exists a constant $C$ such that

$$
\left\|\partial^{\alpha} f\right\|_{p(\cdot)} \leq C\|f\|_{p(\cdot)}
$$

holds for all $f \in L_{p(\cdot)}^{K}$.

Proof. See [18, Theorems 3.5/2, 3.5/5].

Theorem 3.5. Let $\Omega$ be an open set in $\mathbb{R}^{n}$ and let $p(\cdot) \in \mathscr{P}^{0}$.

1. $C_{0}^{\infty}(\Omega) \hookrightarrow \mathscr{B}_{p(\cdot)}^{c}(\Omega)$ and $C_{0}^{\infty}(\Omega)$ is sequentially dense in $\mathscr{B}_{p(\cdot)}^{c}(\Omega)$.

2. If $M$ is bounded in $L_{p(\cdot) / p_{0}}$ for some $0<p_{0}<p_{-}$, then $\mathscr{B}_{p(\cdot)}^{c}(\Omega) \hookrightarrow S^{\prime}$. (If $p_{-} \geq 1$, the hypothesis on $M$ is not necessary.)

3. Let $M$ be as in 2. Then the inductive limit $\mathscr{B}_{p(\cdot)}^{c}(\Omega)$ is regular (i.e. every bounded set in $\mathscr{B}_{p(\cdot)}^{c}(\Omega)$ is contained and bounded in some step) and complete. (If $p_{-} \geq 1$, the hypothesis on $M$ is not necessary.)

4. Let $M$ as in 2. Let $\varphi \in S$ (resp. P a polynomial in $\left.\mathbb{R}^{n}\right)$. Then the multiplication operator $M_{\varphi}$ (resp. $\left.M_{P}\right)$ is continuous from $\mathscr{B}_{p(\cdot)}^{c}(\Omega)$ into $\mathscr{B}_{p(\cdot)}^{c}(\Omega)$.

5. Assume $n=1$. Let $p(\cdot)$ be such that $1<p_{-} \leq p_{+}<\infty$ and $M$ is bounded in $L_{p(\cdot)}(\mathbb{R})$. Let $\left.\Omega=\right] a, b\left[(-\infty \leq a<b \leq \infty)\right.$. Let $a_{j} \searrow a, b_{j} \nearrow b$, and we put $K_{j}=\left[a_{j}, b_{j}\right]$ for $j=1,2, \ldots$ Then $\mathscr{B}_{p(\cdot)}^{c}(\Omega) \simeq L_{p(\cdot)}^{-K_{1}} \oplus G_{1} \oplus G_{2} \oplus \cdots$ being each $G_{j}$ an infinite dimensional complemented subspace of $L_{p(\cdot)}^{-K_{j+1}}$. In the case $p(\cdot) \equiv p$, the space $\mathscr{B}_{p(\cdot)}^{c}(\Omega)$ is isomorphic to $\left(l_{p}(\mathbb{Z})\right)^{(\mathbb{N})}$. Finally, if $p(\cdot) \equiv$ 
$p$ and $0<p<1$ then the Banach envelope of each step $\mathscr{B}_{p(\cdot)} \cap \mathscr{E}^{\prime}\left(K_{j}\right)$ is isomorphic to $l_{1}$.

\section{Proof.}

1. It is easily seen that the natural mapping $C_{0}^{\infty}(\Omega) \rightarrow \mathscr{B}_{p(\cdot)}^{c}(\Omega): \varphi \rightarrow\langle\theta, \varphi\rangle=$ $\int_{\mathbb{R}^{n}} \theta \varphi d x(\theta \in S)$ is well defined and is linear and injective. Next we prove that it is continuous. If $K$ is any compact subset of $\Omega$ then $\langle\cdot, \varphi\rangle \in \mathscr{B}_{p(\cdot)} \cap \mathscr{E}^{\prime}(K)$ for all $\varphi \in C_{0}^{\infty}(K)$. Then if $\varphi_{j} \rightarrow 0$ in $C_{0}^{\infty}(K)$ it results that $\varphi_{j} \rightarrow 0$ in $S$, i.e. $\hat{\varphi}_{j} \rightarrow 0$ in $S$, thus $\hat{\varphi}_{j} \rightarrow 0$ in $L_{p(\cdot)}$ which implies that $\left\langle\cdot, \varphi_{j}\right\rangle \rightarrow 0$ in $\mathscr{B}_{p(\cdot)} \cap \mathscr{E}^{\prime}(K)$. Since $C_{0}^{\infty}(\Omega)$ and $\mathscr{B}_{p(\cdot)}^{c}(\Omega)$ are inductive limits the required continuity is shown. In order to prove the density we take any $u$ in $\mathscr{B}_{p(\cdot)}^{c}(\Omega)$ and we apply Lemma 3.3. Then, $\theta_{\varepsilon} * u \rightarrow u$ in $\mathscr{B}_{p(\cdot)}$ (without loss of generality, we can assume that $\left.\operatorname{supp} \theta \subset B_{1}\right)$. If $u \in \mathscr{B}_{p(\cdot)} \cap \mathscr{E}^{\prime}(K)$ and $K+B_{\varepsilon_{0}} \subset \Omega$ then $\left(\theta_{\varepsilon} * u\right)_{\varepsilon \leq \varepsilon_{0}} \subset C_{0}^{\infty}(\Omega)$ and $\theta_{\varepsilon} * u \rightarrow u$ in $\mathscr{B}_{p(\cdot)} \cap \mathscr{E}^{\prime}\left(K+B_{\varepsilon_{0}}\right)$, therefore $\theta_{\varepsilon} * u \rightarrow u$ in $\mathscr{B}_{p(\cdot)}^{c}(\Omega)$.

2. Let $K$ be any compact subset of $\Omega$ and consider the following diagram

$$
\mathscr{B}_{p(\cdot)} \cap \mathscr{E}^{\prime}(K) \stackrel{\mathscr{F}}{\rightarrow} L_{p(\cdot)}^{-K} \stackrel{j}{\rightarrow} S^{\prime} \stackrel{\mathscr{F}^{-1}}{\longrightarrow} S^{\prime}
$$

where $\mathscr{F}$ is the Fourier transform, $j$ is the canonical injection and $\mathscr{F}^{-1}$ is the inverse Fourier transform. Since $\mathscr{F}$ is an isomorphism, $j$ is continuous (use the hypothesis on $M$ and [18, Theorem 3.5/4]) and $\mathscr{F}^{-1}$ is an automorphism, it results that the canonical injection $\mathscr{B}_{p(\cdot)} \cap \mathscr{E}^{\prime}(K) \rightarrow S^{\prime}$ is also continuous. In consequence, $\mathscr{B}_{p(\cdot)}^{c}(\Omega) \hookrightarrow S^{\prime}$.

3. It is easy to see that $\mathscr{B}_{p(\cdot)}^{c}(\Omega)$ coincides with the inductive limit ind ${ }_{j}\left[\mathscr{B}_{p(\cdot)} \cap\right.$ $\left.\mathscr{E}^{\prime}\left(K_{j}\right)\right]$ where $\left\{K_{j}\right\}_{j=1}^{\infty}$ is any fundamental sequence of compact subsets of $\Omega$. Then, since $M$ is bounded in $L_{p(\cdot) / p_{0}}$, it follows from [18, Theorem 3.5/4] that each $L_{p(\cdot)}^{-K_{j}}$ is complete (i.e. a quasi-Banach space). Thus each step $\mathscr{B}_{p(\cdot)} \cap$ $\mathscr{E}^{\prime}\left(K_{j}\right)$ is also complete and so $\mathscr{B}_{p(\cdot)} \cap \mathscr{E}^{\prime}\left(K_{j}\right)$ is closed in $\mathscr{B}_{p(\cdot)} \cap \mathscr{E}^{\prime}\left(K_{j+1}\right)$ for all $j$. In view of [9, Theorem 2 p. 84, Theorem 4 p. 86], the inductive limit $\mathscr{B}_{p(\cdot)}^{c}(\Omega)$ is regular and complete.

4. If $K$ is any compact subset of $\Omega$, it is sufficient to show that $M_{\varphi}$ and $M_{P}$ are bounded operators from $\mathscr{B}_{p(\cdot)} \cap \mathscr{E}^{\prime}(K)$ into $\mathscr{B}_{p(\cdot)} \cap \mathscr{E}^{\prime}(K)$. But this is a consequence of the following commutative diagrams and Lemma 3.4:
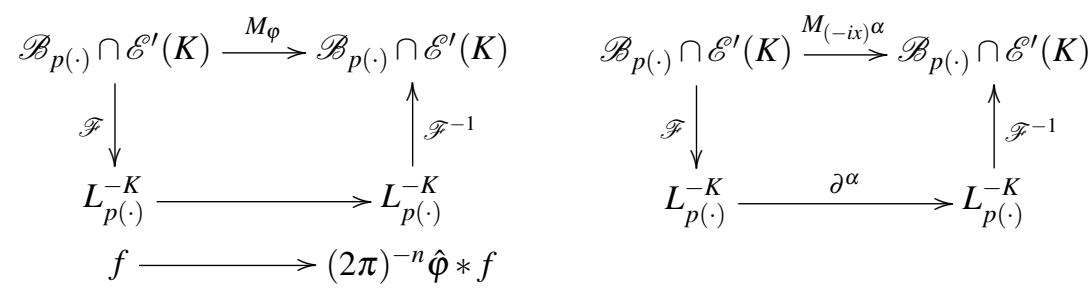

5. We have $\mathscr{B}_{p(\cdot)}^{c}(\Omega)=\operatorname{ind}_{j}\left[\mathscr{B}_{p(\cdot)} \cap \mathscr{E}^{\prime}\left(K_{j}\right)\right]$ and here each step $\mathscr{B}_{p(\cdot)} \cap \mathscr{E}^{\prime}\left(K_{j}\right)$ is isomorphic (via Fourier transform) to $L_{p(\cdot)}^{-K_{j}}$. On the other hand, by [2, Theorem 8.14], the Hilbert transform is bounded in $L_{p(\cdot)}(\mathbb{R})$. Hence it follows, 
reasoning as in the classical case, that every $\chi_{-K_{j}}$ is an $L_{p(\cdot)}(\mathbb{R})$-Fourier multiplier (i.e. the operator associated with $\chi_{-K_{j}}, S_{-K_{j}}(f)=\mathscr{F}^{-1}\left(\chi_{-K_{j}} \hat{f}\right)$, is bounded in $\left.L_{p(\cdot)}(\mathbb{R})\right)$. Then, since $L_{p(\cdot)}(\mathbb{R})=L_{p(\cdot)}^{-K_{j}} \oplus \operatorname{ker} S_{-K_{j}}$, we get $L_{p(\cdot)}^{-K_{j+1}}=$ $L_{p(\cdot)}^{-K_{j}} \oplus\left(\operatorname{ker} S_{-K_{j}} \cap L_{p(\cdot)}^{-K_{j+1}}\right)$ which shows that $G_{j}=\operatorname{ker} S_{-K_{j}} \cap L_{p(\cdot)}^{-K_{j+1}}$ is an infinite-dimensional (if $Q$ is a compact interval such that $Q \subset-K_{j+1} \backslash\left(-K_{j}+\right.$ $[-\varepsilon, \varepsilon])$, for a sufficiently small $\varepsilon>0$, then $G_{j} \supset S^{Q}$ ) topological complement of $L_{p(\cdot)}^{-K_{j}}$ in $L_{p(\cdot)}^{-K_{j+1}}$. Next, using the fact previous to Lemma 3.3, we obtain the required isomorphism: $\mathscr{B}_{p(\cdot)}^{c}(\Omega) \simeq L_{p(\cdot)}^{-K_{1}} \oplus G_{1} \oplus G_{2} \oplus \cdots$. If $p(\cdot) \equiv p$ we know (see e.g. [22, pp. 239,240]) that every $L_{p}^{-K_{j}}$ is isomorphic to $l_{p}(\mathbb{Z})$, thus $G_{j}$ is isomorphic to an infinite-dimensional complemented subspace of $l_{p}(\mathbb{Z})$ and so, since the space $l_{p}(\mathbb{Z})$ is prime [12, Theorem 2.a.3], $G_{j}$ becomes isomorphic to $l_{p}(\mathbb{Z})$. We conclude that $\mathscr{B}_{p}^{c}(\Omega) \simeq\left(l_{p}(\mathbb{Z})\right)^{(\mathbb{N})}$ (see also [6], [16]). If $0<p<1$ Hoffmann [7] proved that the Banach envelope of the quasi-Banach space $L_{p}^{[-\pi, \pi]}$ is isomorphic to $l_{1}$, thus our claim is an immediate consequence of this result.

\section{Remark 3.6.}

1. Let us recall that a bounded open set $\Omega$ in $\mathbb{R}^{n}$ has the segment property if there exist open balls $V_{j}$ and vectors $y^{j} \in \mathbb{R}^{n}, j=1, \ldots, N$, such that $\bar{\Omega} \subset \cup_{j=1}^{N} V_{j}$ and $\left(\bar{\Omega} \cap V_{j}\right)+t y^{j} \subset \Omega$ for $0<t<1$ and $j=1, \ldots, N$. In [18, Theorem 3.5/3] it is shown that if $M$ is bounded in $L_{p(\cdot) / p_{0}}$ for some $0<p_{0}<p_{-}$and $K=\bar{O}$, being $O$ a bounded open set with the segment property, then $S^{K}$ is dense in $L_{p(\cdot)}^{K}$. By using this result, it is immediate to check that $C_{0}^{\infty}(K)$ is also dense in $\mathscr{B}_{p(\cdot)} \cap \mathscr{E}^{\prime}(K)(p(\cdot)$ and $K$ as before). This improves Theorem 3.5/1 (versus the additional hypothesis that $M$ is bounded in $L_{p(\cdot) / p_{0}}$ for some $0<p_{0}<p_{-}$) since all open set in $\mathbb{R}^{n}$ has a fundamental sequence of compact subsets which are the closures of open sets with the segment property.

2. In [8, Chapter $X V, 15.2]$ Hörmander obtains a family of seminorms defining the inductive limit topology of $\mathscr{B}_{2, k}^{c}(\Omega)$ when $k$ is a Hörmander weight. In this note we extend this result to the variable exponent Hörmander spaces $\mathscr{B}_{p(\cdot)}^{c}(\Omega)$ when $p(\cdot)$ is as in Lemma 3.4 and $p_{-} \geq 1$ : If $\left(\theta_{j}\right)_{j=1}^{\infty}$ is a $C_{0}^{\infty}(\Omega)$-partition of unity on $\Omega$, then the inductive limit topology of $\mathscr{B}_{p(\cdot)}^{c}(\Omega)$ is generated by the system of seminorms

$$
\|u\|_{\left(C_{i}\right)}:=\sum_{i=1}^{\infty} C_{i}\left\|\theta_{i} u\right\|_{\mathscr{B}_{p(\cdot)}}, \quad u \in \mathscr{B}_{p(\cdot)}^{c}(\Omega),\left(C_{i}\right)_{i=1}^{\infty} \in\left(\mathbb{R}_{+}\right)^{\mathbb{N}}
$$

Let $\left\{K_{j}\right\}_{j=1}^{\infty}$ be a fundamental sequence of compact subsets of $\Omega$. For each $u \in \mathscr{B}_{p(\cdot)}^{c}(\Omega), \theta_{i} u=0$ for all $i$ large enough. Indeed, if supp $u \subset K_{j}$ there exist a relatively compact open set $W_{j}$ and a positive integer $m_{j}$ such that $K_{j} \subset W_{j} \subset \bar{W}_{j} \subset \Omega$ and $\theta_{1}(x)+\cdots+\theta_{m_{j}}(x)=1$ in $W_{j}$. Therefore $\theta_{i}=0$ in $W_{j}$ for $i>m_{j}$ and so $\theta_{i} u=0$ for these indexes. Consequently, $u=\sum_{i=1}^{m_{j}} \theta_{i} u$. Hence 
it follows that, for each $\left(C_{i}\right)_{i=1}^{\infty} \in\left(\mathbb{R}_{+}\right)^{\mathbb{N}}$, the mapping $\mathscr{B}_{p(\cdot)}^{c}(\Omega) \rightarrow[0, \infty[: u \rightarrow$ $\|u\|_{\left(C_{i}\right)}$ is a seminorm on $\mathscr{B}_{p(\cdot)}^{c}(\Omega)$. Let $\mathscr{T}$ be the topology generated by these seminorms. If $\left(C_{i}\right)_{i=1}^{\infty} \in\left(\mathbb{R}_{+}\right)^{\mathbb{N}}$, the Lemma $3.4 / 1$ and the continuity of the Fourier transform show that exist a positive integer $k$ and a positive constant $C$ such that

$$
\begin{aligned}
\|u\|_{\left(C_{i}\right)}=\sum_{i=1}^{m_{j}} C_{i}\left\|\theta_{i} u\right\|_{\mathscr{B}_{p(\cdot)}}=\sum_{i=1}^{m_{j}} C_{i}\left\|\widehat{\theta_{i} u}\right\|_{p(\cdot)}=(2 \pi)^{-n} \sum_{i=1}^{m_{j}} C_{i}\left\|\hat{\theta}_{i} * \hat{u}\right\|_{p(\cdot)} \\
\leq C\left(\sum_{i=1}^{m_{j}} C_{i}\left|\theta_{i}\right|_{k}\right)\|u\|_{\mathscr{B}_{p(\cdot)}}
\end{aligned}
$$

holds for all $u \in \mathscr{B}_{p(\cdot)} \cap \mathscr{E}^{\prime}\left(K_{j}\right)$. This proves the continuity of the canonical injection $\mathscr{B}_{p(\cdot)} \cap \mathscr{E}^{\prime}\left(K_{j}\right) \rightarrow \mathscr{B}_{p(\cdot)}^{c}(\Omega)[\mathscr{T}]$. Since this is valid for all $j$, the topology $\mathscr{T}$ is coarser than the inductive limit topology. Let us see the reverse inclusion: Let $\left\{K_{j_{l}}\right\}_{l=1}^{\infty}$ be a subsequence of $\left\{K_{j}\right\}_{j=1}^{\infty}$ such that $\operatorname{supp} \theta_{l} \subset K_{j_{l}}$ for all $l$. If $\|\cdot\|$ is a continuous seminorm in the space $\mathscr{B}_{p(\cdot)}^{c}(\Omega)$ equipped with the inductive limit topology, its restriction to each step is continuous. So there are constants $C_{l}>0$ such that $\|u\| \leq C_{l}\|u\|_{\mathscr{B}_{p(\cdot)}}$ for all $u \in \mathscr{B}_{p(\cdot)} \cap \mathscr{E}^{\prime}\left(K_{j_{l}}\right)$ $l=1,2, \ldots$ Let $u \in \mathscr{B}_{p(\cdot)}^{c}(\Omega)$. If $\operatorname{supp} u \subset K_{j}$, we know that $u=\sum_{i=1}^{m_{j}} \theta_{i} u$ for some positive integer $m_{j}$. Since each $\theta_{i} u$ is in $\mathscr{B}_{p(\cdot)} \cap \mathscr{E}^{\prime}\left(K_{j_{i}}\right)$, we obtain

$$
\|u\| \leq \sum_{i=1}^{m_{j}}\left\|\theta_{i} u\right\| \leq \sum_{i=1}^{m_{j}} C_{i}\left\|\theta_{i} u\right\|_{\mathscr{B}_{p(\cdot)}}=\sum_{i=1}^{\infty} C_{i}\left\|\theta_{i} u\right\|_{\mathscr{B}_{p(\cdot)}}=\|u\|_{\left(C_{i}\right)} .
$$

In consequence the two topologies coincide.

In the next theorem we show a number of basic properties of the spaces $\mathscr{B}_{p(\cdot)}^{\text {loc }}(\Omega)$ that we shall need to study the duality $\left\langle\mathscr{B}_{p(\cdot)}^{c}(\Omega), \mathscr{B}_{p^{\prime}(\cdot)}^{\text {loc }}(\Omega)\right\rangle$.

Theorem 3.7. Let $\Omega$ be an open set in $\mathbb{R}^{n}$ and let $p(\cdot) \in \mathscr{P}^{0}$ be such that $M$ is bounded in $L_{p(\cdot) / p_{0}}$ for some $0<p_{0}<p_{-}$. Then:

1. $\mathscr{B}_{p(\cdot)}^{\text {loc }}(\Omega)$ is a metrizable topological linear space,

2. We have the natural embeddings $C^{\infty}(\Omega) \hookrightarrow \mathscr{B}_{p(\cdot)}^{\text {loc }}(\Omega) \hookrightarrow \mathscr{D}^{\prime}(\Omega), C_{0}^{\infty}(\Omega) \stackrel{d}{\hookrightarrow}$ $\mathscr{B}_{p(\cdot)}^{\text {loc }}(\Omega)$,

3. $\mathscr{B}_{p(\cdot)}^{\text {loc }}(\Omega)$ is complete (i.e. an F-space; a Fréchet space if $p_{-} \geq 1$ ).

\section{Proof.}

1. Let $u \in \mathscr{B}_{p(\cdot)}^{\text {loc }}(\Omega) \backslash\{0\}$ and let $\varphi \in C_{0}^{\infty}(\Omega)$ such that $\langle\varphi, u\rangle \neq 0$. Then $\|u\|_{p(\cdot), \theta}$ $>0$ for all $\theta \in C_{0}^{\infty}(\Omega)$ such that $\theta=1$ in $\operatorname{supp} \varphi$. Thus the topology of $\mathscr{B}_{p(\cdot)}^{\text {loc }}(\Omega)$ is Hausdorff. We now consider a fundamental sequence $\left\{K_{j}\right\}_{j=1}^{\infty}$ of compact subsets of $\Omega$ and choose $\varphi_{j} \in C_{0}^{\infty}(\Omega)$ such that $\varphi_{j} \equiv 1$ on $K_{j}$ and $\operatorname{supp} \varphi_{j} \subset$ $\stackrel{\circ}{K}_{j+1}, j=1,2, \ldots$ Then the topology of $\mathscr{B}_{p(\cdot)}^{\text {loc }}(\Omega)$ is also generated by the system of semiquasi-norms $\left\{\|\cdot\|_{p(\cdot), \varphi_{j}}: j=1,2, \ldots\right\}$. In order to prove this, we 
choose a function $\varphi \in C_{0}^{\infty}(\Omega)$ and an integer $j$ such that $\operatorname{supp} \varphi \subset K_{j}$. Then, for each $u \in \mathscr{B}_{p(\cdot)}^{\text {loc }}(\Omega)$, we have $\varphi u=\varphi\left(\varphi_{j} u\right)$ where $\varphi_{j} u \in \mathscr{B}_{p(\cdot)} \cap \mathscr{E}^{\prime}\left(K_{j+1}\right)$. Thus, by using Lemma 3.4/1 and the continuity in $S$ of the Fourier transform, we get a positive constant $C$ and a positive integer $m$ such that

$$
\begin{aligned}
\|u\|_{p(\cdot), \varphi}=\|\varphi u\|_{\mathscr{B}_{p(\cdot)}}=\left\|\varphi\left(\varphi_{j} u\right)\right\|_{\mathscr{B}_{p(\cdot)}} & =\left\|\widehat{\varphi\left(\varphi_{j} u\right)}\right\|_{p(\cdot)}=(2 \pi)^{-n}\left\|\hat{\varphi} * \widehat{\varphi_{j} u}\right\|_{p(\cdot)} \\
& \leq C|\varphi|_{m}\left\|\varphi_{j} u\right\|_{\mathscr{B}_{p(\cdot)}}=C|\varphi|_{m}\|u\|_{p(\cdot), \varphi_{j}}
\end{aligned}
$$

holds for all $u \in \mathscr{B}_{p(\cdot)}^{\text {loc }}(\Omega)$. This proves 1 .

2. We will only show the density of $C_{0}^{\infty}(\Omega)$ in $\mathscr{B}_{p(\cdot)}^{\text {loc }}(\Omega)$. Let $u \in \mathscr{B}_{p(\cdot)}^{\text {loc }}(\Omega)$. Given $\varphi \in C_{0}^{\infty}(\Omega)$ and $\varepsilon>0$ we have to prove that there exists a function $\phi \in C_{0}^{\infty}(\Omega)$ such that $\|u-\phi\|_{p(\cdot), \varphi}<\varepsilon$. Let $\psi \in C_{0}^{\infty}(\Omega)$ so that $\psi=1$ in supp $\varphi$. Then $\psi u \in \mathscr{B}_{p(\cdot)}^{c}(\Omega)$, and there exists a sequence $\left(\chi_{v}\right)$ in $C_{0}^{\infty}(\Omega)$ such that $\chi_{v} \rightarrow \psi u$ in $\mathscr{B}_{p(\cdot)}^{c}(\Omega)$ when $v \rightarrow \infty$ (apply Theorem 3.5/1). Hence and from Theorem 3.5/4 if follows that $\varphi \chi_{v} \rightarrow \varphi(\psi u)=\varphi u$ in $\mathscr{B}_{p(\cdot)}^{c}(\Omega)$ and, a fortiori, in $\mathscr{B}_{p(\cdot)}$. Therefore, putting $\phi=\chi_{v}$ with $v$ sufficiently large, we have $\|u-\phi\|_{p(\cdot), \varphi}<\varepsilon$.

3. Let $\left(u_{j}\right)_{j=1}^{\infty}$ be a Cauchy sequence in $\mathscr{B}_{p(\cdot)}^{\text {loc }}(\Omega)$ (only consider sequences in virtue of 1). By 2 and the completeness of $\mathscr{D}^{\prime}(\Omega), u_{j}$ has a limit $u$ in $\mathscr{D}^{\prime}(\Omega)$. Let us see that $u \in \mathscr{B}_{p(\cdot)}^{\text {loc }}(\Omega)$. Let $\varphi \in C_{0}^{\infty}(\Omega)$. Obviously, $\varphi u_{j} \rightarrow \varphi u$ in $\mathscr{D}^{\prime}(\Omega)$. Furthermore, $\left(\varphi u_{j}\right)_{j=1}^{\infty}$ is a Cauchy sequence in the quasi-Banach space $\mathscr{B}_{p(\cdot)}$ $\cap \mathscr{E}^{\prime}(\operatorname{supp} \varphi)$. Let $v$ be the limit of $\varphi u_{j}$ in this space. From Theorem 3.5/2 we conclude that $\varphi u=v$. Hence it follows that $u \in \mathscr{B}_{p(\cdot)}^{\text {loc }}(\Omega)$ and that $u_{j} \rightarrow u$ in $\mathscr{B}_{p(\cdot)}^{\text {loc }}(\Omega)$.

\section{The dual of $\mathscr{B}_{p(\cdot)}^{c}(\Omega)$}

In [8, Chapter XV], Hörmander studies the behaviour of the Fourier-Laplace transform in the space $\mathscr{B}_{2, k}^{c}(\Omega)=\operatorname{ind}_{K}\left[\mathscr{B}_{2, k} \cap \mathscr{E}^{\prime}(K)\right]$ when $\Omega$ is an open convex set in $\mathbb{R}^{n}$ and $k$ satisfies the estimate $k(x+y) \leq(1+C|x|)^{N} k(y), x, y \in \mathbb{R}^{n}(C$ and $N$ positive constants). For this he analyses the inductive topology in $\mathscr{B}_{2, k}^{c}(\Omega)$, proves the isomorphism $\left(\mathscr{B}_{2, k}^{c}(\Omega)\right)^{\prime} \simeq \mathscr{B}_{2,1 / \tilde{k}}^{\text {loc }}(\Omega)$ and shows that every continuous seminorm in $\mathscr{B}_{2, k}^{c}(\Omega)$ is bounded by a seminorm of the form $u \rightarrow\left(\int_{\mathbb{C}^{n}}|\hat{u}(\zeta)|^{2} e^{-2 \phi(\zeta)} d \lambda(\zeta)\right)^{1 / 2}$, where $\hat{u}$ is the Fourier-Laplace transform of $u$ and $\phi$ is plurisubharmonic (see [8, Section 15.2]). In [16, Section 3] the former isomorphism is extended to Hörmander spaces in the sense of Beurling and Björck. A number of applications of this duality (to sequence space representations of several ultradistributions spaces and to linear partial differential operators) are also given in [16] and [17]. In this section we extend the former isomorphism to variable exponent Hörmander spaces. As a consequence, some results on sequence space representations of variable exponent 
Hörmander spaces are given. Finally, we propose a question on interpolation of the spaces $\mathscr{B}_{p(\cdot)} \cap \mathscr{E}^{\prime}(K)$.

Lemma 4.1. Let $p \in \mathscr{P}^{0}$ be with $p_{-}>1$ and let $f$ be a measurable function on $\mathbb{R}^{n}$ such that $f \varphi \in L_{1}$ for all $\varphi \in S$ and such that $B:=\sup \left\{\left|\int_{\mathbb{R}^{n}} f \varphi d x\right|: \varphi \in\right.$ $\left.S,\|\varphi\|_{p^{\prime}(\cdot)} \leq 1\right\}<\infty$. Then $f \in L_{p(\cdot)}$.

Proof. We define the functional $u(\varphi):=\int_{\mathbb{R}^{n}} f \varphi d x, \varphi \in S$. Since $|u(\varphi)| \leq B\|\varphi\|_{p^{\prime}(\cdot)}$ for all $\varphi \in S$, it follows that $u \in\left(S,\|\cdot\|_{p^{\prime}(\cdot)}\right)^{\prime}$ and, since $S$ is dense in $L_{p^{\prime}(\cdot)}$ (use Lemma 2.4/1), $u$ has a unique continuous linear extension $U$ to $L_{p^{\prime}(\cdot)}$. Next, by duality (Lemma 2.3), we can find a function $g \in L_{p(\cdot)}$ such that $\int_{\mathbb{R}^{n}} g \varphi d x=U(\varphi)=$ $\int_{\mathbb{R}^{n}} f \varphi d x$ for all $\varphi \in S$. In consequence, $f=g$ and $f \in L_{p(\cdot)}$.

Lemma 4.2. Let $p(\cdot) \in \mathscr{P}^{0}$ be such that $p_{-}>1$ and such that $M$ is bounded in $L_{p(\cdot)}$. Let $K$ be a locally integrable function in $\mathbb{R}^{n} \backslash\{0\}$ such that $\widehat{K} \in L_{\infty},|K(x)| \leq$ $C|x|^{-n}$ and $|\nabla K(x)| \leq C|x|^{-(n+1)}$ for all $x \neq 0$. Then the singular integral operator $T$, defined by $T f(x)=K * f(x)$, is bounded in $L_{p(\cdot)}$.

Proof. See [1, p. 247].

Theorem 4.3. Let $\Omega$ be an open set in $\mathbb{R}^{n}$ and let $p(\cdot) \in \mathscr{P}^{0}$ be such that $p_{-}>1$ and $M$ is bounded in $L_{p(\cdot)}$. Then $\left(\mathscr{B}_{p(\cdot)}^{c}(\Omega)\right)^{\prime}$ is isomorphic to $\mathscr{B}_{p^{\prime}(\cdot)}^{\text {loc }}(\Omega)$.

Proof. Let $J$ be the natural embedding

$$
C_{0}^{\infty}(\Omega) \rightarrow \mathscr{B}_{p(\cdot)}^{c}(\Omega): \varphi \rightarrow\langle\theta, J(\varphi)\rangle=\int_{\mathbb{R}^{n}} \theta \varphi d x \quad(\theta \in S)
$$

(see Theorem 3.5/1) and consider its adjoint operator $J^{\prime}:\left(\mathscr{B}_{p(\cdot)}^{c}(\Omega)\right)^{\prime} \rightarrow \mathscr{D}^{\prime}(\Omega)$. Let us see that $\operatorname{Im} J^{\prime} \subset \mathscr{B}_{p^{\prime}(\cdot)}^{\text {loc }}(\Omega)$. Let $l \in\left(\mathscr{B}_{p(\cdot)}^{c}(\Omega)\right)^{\prime}$. We have to show that $\varphi J^{\prime}(l) \in$ $\mathscr{B} \widetilde{p^{\prime}(\cdot)}$, i.e. $\mathscr{F}\left(\varphi J^{\prime}(l)\right) \in L_{p^{\prime}(\cdot)}$, for all $\varphi \in C_{0}^{\infty}(\Omega)$. Let us fix such a $\varphi$ and set $K=$ $\operatorname{supp} \varphi$. Then $\mathscr{F}^{2}\left(\varphi J^{\prime}(l)\right)=(2 \pi)^{n} \widetilde{\varphi J^{\prime}(l)}=(2 \pi)^{n} \hat{\varphi} \widetilde{J^{\prime}(l)}$ and so supp $\mathscr{F}^{2}\left(\varphi J^{\prime}(l)\right) \subset$ $-K$. This implies, by the Paley-Wiener-Schwartz theorem, that $\mathscr{F}\left(\varphi J^{\prime}(l)\right)$ is an entire analytic function of $n$ complex variables such that for any $\varepsilon>0$

$$
\left|\mathscr{F}\left(\varphi J^{\prime}(l)\right)(z)\right| \leq A_{\mathcal{E}}(1+|x|)^{\lambda} e^{(\sigma+\varepsilon)|y|}
$$

holds for all $z=x+i y$ with $x, y \in \mathbb{R}^{n}\left(\lambda \in \mathbb{R}\right.$ is a constant and $A_{\varepsilon}$ depends on $\varepsilon$ but not on $z$ ) (see e.g. [21, p. 272]). We now prove that $\sup \left\{\left|\int_{\mathbb{R}^{n}} \mathscr{F}\left(\varphi J^{\prime}(l)\right) \theta d x\right|: \theta \in\right.$ $\left.S,\|\theta\|_{\widetilde{p(\cdot)}} \leq 1\right\}<\infty$ since, once this is established, Lemma 4.1 yields $\mathscr{F}\left(\varphi J^{\prime}(l)\right) \in$ $L_{\widetilde{p^{\prime}(\cdot)}}$. Fix an element $\theta \in S$ such that $\|\theta\|_{\widetilde{p(\cdot)}} \leq 1$. Then, from the continuity of $l$ on the step $\mathscr{B}_{p(\cdot)} \cap \mathscr{E}^{\prime}(K)$ and from the continuity in $L_{p(\cdot)}$ of the operator $\theta \rightarrow \theta * \hat{\varphi}$ (Lemma 4.2), we get

$$
\begin{array}{r}
\left|\int_{\mathbb{R}^{n}} \mathscr{F}\left(\varphi J^{\prime}(l)\right) \theta d x\right|=\left|\left\langle\theta, \mathscr{F}\left(\varphi J^{\prime}(l)\right)\right\rangle\right|=|\langle J(\hat{\theta} \varphi), l\rangle| \leq C\|J(\hat{\theta} \varphi)\|_{\mathscr{B}_{p(\cdot)}} \\
=C\|\mathscr{F}(\hat{\theta} \varphi)\|_{p(\cdot)}=C\|\tilde{\theta} * \hat{\varphi}\|_{p(\cdot)} \leq C\|\tilde{\theta}\|_{p(\cdot)}=C\|\theta\|_{\widetilde{p(\cdot)}} \leq C .
\end{array}
$$


Therefore, we have shown that $\operatorname{Im} J^{\prime} \subset \mathscr{B}_{p^{\prime}(\cdot)}^{\text {loc }}(\Omega)$. Next we prove that the mapping

$$
J^{\prime}:\left(\mathscr{B}_{p(\cdot)}^{c}(\Omega)\right)^{\prime} \rightarrow \underset{p^{\prime}}{\text { loc }}(\Omega)
$$

is onto: Let $\left\{K_{j}\right\}_{j=1}^{\infty}$ be a fundamental sequence of compact subsets of $\Omega$ such that every $K_{j}$ is the closure of an open set with the segment property; moreover, let $\chi_{j} \in C_{0}^{\infty}(\Omega)$ be such that $\chi_{j}(x)=1$ whenever $x \in K_{j}, j=1,2, \ldots$ Let $u \in \mathscr{B} \frac{\operatorname{loc}}{p^{\prime}(\cdot)}(\Omega)$. For each $j$ we define $l_{j}$ by $\left\langle J(\varphi), l_{j}\right\rangle:=\langle\varphi, u\rangle$ for all $\varphi \in C_{0}^{\infty}\left(K_{j}\right)$. Then, taking into account the generalized Hölder inequality, we get

$$
\begin{aligned}
\left|\left\langle J(\varphi), l_{j}\right\rangle\right|=|\langle\varphi, u\rangle|=\left|\left\langle\varphi, \chi_{j} u\right\rangle\right|=C\left|\int_{\mathbb{R}^{n}} \widehat{\chi_{j} u} \tilde{\hat{\varphi}} d x\right| \\
\quad \leq C\|\tilde{\hat{\varphi}}\|_{\widetilde{p(\cdot)}}\left\|\widehat{\chi_{j} u}\right\|_{\left(\widetilde{p(\cdot))^{\prime}}\right.}=C\|\hat{\varphi}\|_{p(\cdot)}=C\|J(\varphi)\|_{\mathscr{B}_{p(\cdot)}}
\end{aligned}
$$

for all $\varphi \in C_{0}^{\infty}\left(K_{j}\right)$. Hence and from the density of $J\left(C_{0}^{\infty}\left(K_{j}\right)\right)$ in the step $\mathscr{B}_{p(\cdot)} \cap$ $\mathscr{E}^{\prime}\left(K_{j}\right)$ (use Lemma $2.4 / 1$ and Remark 3.6/1) it follows that $l_{j}$ extends to a unique continuous linear form $\bar{l}_{j}$ on $\mathscr{B}_{p(\cdot)} \cap \mathscr{E}^{\prime}\left(K_{j}\right)$. Finally, since $\bar{l}_{j+1}$ and $\bar{l}_{j}$ coincide on $\mathscr{B}_{p(\cdot)} \cap \mathscr{E}^{\prime}\left(K_{j}\right)$, we easily obtain an $l$ in $\left(\mathscr{B}_{p(\cdot)}^{c}(\Omega)\right)^{\prime}$ with $J^{\prime}(l)=u$. To sum up, $J^{\prime}$ is an algebraic isomorphism from $\left(\mathscr{B}_{p(\cdot)}^{c}(\Omega)\right)^{\prime}$ onto $\mathscr{B}_{p^{\prime}(\cdot)}^{\text {loc }}(\Omega)$. To see that $J^{\prime}$ is a (topological) isomorphism it suffices to prove that it is continuous since that those spaces are Fréchet spaces $\left(\mathscr{B} \frac{\text { loc }}{p^{\prime}(\cdot)}(\Omega)\right.$ is a Fréchet space by Lemma 2.4/1 and Theorem $3.7 / 3 ; \mathscr{B}_{p(\cdot)}^{c}(\Omega)$ is a (DF)-space by Theorem $3.5 / 3$ and $[10$, (4) p. 402] and so its strong dual is also a Fréchet space (see [10, (1) p. 397])). Suppose that $l_{v} \rightarrow 0$ in $\left(\mathscr{B}_{p(\cdot)}^{c}(\Omega)\right)^{\prime}$. Fix $\varphi$ in $C_{0}^{\infty}(\Omega)$. Then, taking into account Lemma 2.3 and Lemma 2.5/2, we get

$$
\begin{aligned}
& \left\|\varphi J^{\prime}\left(l_{v}\right)\right\|_{\mathscr{B}_{p^{\prime}(\cdot)}}=\left\|\mathscr{F}\left(\varphi J^{\prime}\left(l_{v}\right)\right)\right\|_{\widetilde{p^{\prime}(\cdot)}} \\
& \leq C \sup \left\{\left|\int_{\mathbb{R}^{n}} \mathscr{F}\left(\varphi J^{\prime}\left(l_{v}\right)\right) \theta d x\right|: \theta \in S,\|\theta\|_{\widetilde{p(\cdot)}} \leq 1\right\} \\
& \quad=C \sup \left\{\left|\left\langle J(\hat{\theta} \varphi), l_{v}\right\rangle\right|: \theta \in S,\|\theta\|_{\widetilde{p(\cdot)}} \leq 1\right\} .
\end{aligned}
$$

But the set $A=\left\{J(\hat{\theta} \varphi): \theta \in S,\|\theta\|_{\widetilde{p(\cdot)}} \leq 1\right\}$ is bounded in $\mathscr{B}_{p(\cdot)}^{c}(\Omega)$ (in fact, if $K=\operatorname{supp} \varphi$ then $A \subset \mathscr{B}_{p(\cdot)} \cap \mathscr{E}^{\prime}(K)$ and so, reasoning as in the first part of the proof, it results that $\sup \left\{\|J(\hat{\theta} \varphi)\|_{\mathscr{B}_{p(\cdot)}}: \theta \in S,\|\theta\|_{\widetilde{p(\cdot)}} \leq 1\right\}<\infty$, but this shows that $A$ is bounded in that step and thus in $\left.\mathscr{B}_{p(\cdot)}^{c}(\Omega)\right)$ which implies, in virtue of the previous estimate and of the convergence to 0 in $\left(\mathscr{B}_{p(\cdot)}^{c}(\Omega)\right)^{\prime}$ of $\left(l_{v}\right)$, that

$$
\left\|\varphi J^{\prime}\left(l_{v}\right)\right\|_{\mathscr{B}}{ }_{p^{\prime}(\cdot)} \leq C \sup _{u \in A}\left|\left\langle u, l_{v}\right\rangle\right| \rightarrow 0
$$

when $v \rightarrow \infty$. Since $\varphi$ is arbitrary, we have shown that $J^{\prime}\left(l_{v}\right) \rightarrow 0$ in $\underset{B^{\prime}}{p^{\prime}(\cdot)}(\Omega)$.

Remark 4.4. "If $E$ is the inductive limit of an increasing sequence $E_{1}\left[\mathscr{T}_{1}\right] \subset E_{2}\left[\mathscr{T}_{2}\right] \subset$ .. of quasi-Banach spaces such that $\mathscr{T}_{n+1}$ induces on $E_{n}$ the topology $\mathscr{T}_{n}$ and the dual $E_{n}^{\prime}$ of $E_{n}\left[\mathscr{T}_{n}\right]$ separates the points of $E_{n}$ for each $n$, then the (strong) dual $E^{\prime}$ 
is isomorphic to the projective limit of the Banach spaces $E_{n}^{\prime}$ via the natural mapping $E^{\prime} \rightarrow \operatorname{proj}_{n} E_{n}^{\prime}: u \rightarrow\left(\left.u\right|_{E_{n}}\right)_{n=1}^{\infty}$ ". (We shall omit the proof of this simple result.) Hence and from Theorem 3.5/3 it follows that if $\Omega$ is an open set in $\mathbb{R}^{n}$ and $p(\cdot) \in$ $\mathscr{P}^{0}$ is such that $M$ is bounded in $L_{p(\cdot) / p_{0}}$ for some $0<p_{0}<p_{-}$, then $\left(\mathscr{B}_{p(\cdot)}^{c}(\Omega)\right)^{\prime}$ is isomorphic to the projective limit of the Banach spaces $\left(\mathscr{B}_{p(\cdot)} \cap \mathscr{E}^{\prime}\left(K_{j}\right)\right)^{\prime}$ (here $\left\{K_{j}\right\}_{j=1}^{\infty}$ is any fundamental sequence of compact subsets of $\left.\Omega\right)$. In particular, if $\Omega$ is as in Theorem $3.5 / 5$ and $p(\cdot) \equiv p$ with $0<p<1$ then $\left(\mathscr{B}_{p}^{c}(\Omega)\right)^{\prime} \simeq \operatorname{proj}_{j} X_{j}$ where the Banach spaces $X_{j}$ are isomorphic to $l_{\infty}$ (use Theorem 3.5/5 and recall that a quasi-Banach space and its Banach envelope have isomorphic duals (see e.g. [13, Corollary 1])).

The sequence space representation $\mathscr{B}_{1, k}^{\text {loc }}(\Omega) \simeq l_{1}^{\mathbb{N}}$ was established by Vogt in [24] ( $\Omega$ an open set in $\mathbb{R}^{n}$ and $k$ a temperate weight function on $\left.\mathbb{R}^{n}\right)$. In [6] and [16] (see also [15], [17]) the sequence space representations $\mathscr{B}_{p, k}^{\text {loc }}(\Omega) \simeq l_{p}^{\mathbb{N}}, 1<p \leq \infty(\Omega$ open set in $\mathbb{R}^{n}, k$ a temperate weight function on $\mathbb{R}^{n}$ in [6] and $k$ a temperate weight function on $\mathbb{R}^{n}$ with $k^{p}$ in the generalized Muckenhoupt class $A_{p}^{*}$ (see [5, p. 453]) when $p<\infty$ in [16]) were obtained. Next we give a result on function sequence space representation of variable exponent Hörmander spaces.

Corollary 4.5. Let $p(\cdot)$ be such that $1<p_{-} \leq p_{+}<\infty$ and $M$ is bounded in $L_{p(\cdot)}(\mathbb{R})$. Let $\left.\Omega=\right] a, b\left[(-\infty \leq a<b \leq \infty)\right.$. Let $a_{j} \searrow a, b_{j} \nearrow b$, and we put $K_{j}=$ $\left[a_{j}, b_{j}\right]$ for $j=1,2, \ldots$ Then $\mathscr{B}_{p(\cdot)}^{\text {loc }}(\Omega)$ is isomorphic to $L_{p(\cdot)}^{-K_{1}} \times \prod_{j=1}^{\infty} H_{j}$ where each $H_{j}$ is isomorphic to an infinite dimensional complemented subspace of $L_{p(\cdot)}^{-K_{j+1}}$ (thus $\mathscr{B}_{p(\cdot)}^{\operatorname{loc}}(\Omega)$ is isomorphic to a complemented subspace of $\left.\left(L_{p(\cdot)}(\mathbb{R})\right)^{\mathbb{N}}\right)$.

Proof. From Lemma $2.5 / 2$ if follows that $M$ is also bounded in $L_{p^{\prime}(\cdot)}(\mathbb{R})$ (and thus in $L_{p^{\prime}(\cdot)}(\mathbb{R})$ ), then using Theorem 4.3 and Theorem 3.5/5, and taking into account that the dual of a locally convex direct sum of Banach spaces is isomorphic to the product of their duals (see e.g. [10, p. 287]) and that the dual of $\frac{L_{p^{\prime}(\cdot)}^{-K_{j}}}{\text { is isomorphic }}$ to $L_{p(\cdot)}^{K_{j}}[18$, Theorem 4.3], we get the isomorphisms

$$
\mathscr{B}_{p(\cdot)}^{\mathrm{loc}}(\Omega) \simeq\left(\mathscr{B}_{p^{\prime}(\cdot)}^{c}(\Omega)\right)^{\prime} \simeq\left(L_{p^{\prime}(\cdot)}^{-K_{1}} \oplus\left(\oplus_{j=1}^{\infty} G_{j}\right)\right)^{\prime} \simeq L_{p(\cdot)}^{K_{1}} \times \prod_{j=1}^{\infty} G_{j}^{\prime} \simeq L_{p(\cdot)}^{-K_{1}} \times \prod_{j=1}^{\infty} H_{j}
$$

where, for all $j, H_{j}$ is an infinite dimensional complemented subspace of $L_{p(\cdot)}^{-K_{j+1}}$ (the last isomorphism is the operator $\sim$ ).

\section{Some questions}

- It would also be interesting to obtain sequence space representations of the space $\mathscr{B}_{p(\cdot)} \cap \mathscr{E}^{\prime}(Q)\left(Q\right.$ a cube in $\left.\mathbb{R}^{n}\right)$ and of the spaces $\mathscr{B}_{p(\cdot)}^{c}(\Omega)$ and $\mathscr{B}_{p(\cdot)}^{\text {loc }}(\Omega)$ $\left(\Omega\right.$ open set in $\left.\mathbb{R}^{n}\right)$.

- Characterize the variable exponents $p_{0}(\cdot), p_{1}(\cdot)$ (with $1 \leq\left(p_{j}\right)_{-} \leq\left(p_{j}\right)_{+}<\infty$, $j=0,1)$ and the compact subsets $K$ of $\mathbb{R}^{n}$ such that $\left[\mathscr{B}_{p_{0}(\cdot)} \cap \mathscr{E}^{\prime}(K), \mathscr{B}_{p_{1}(\cdot)} \cap\right.$ 
$\left.\mathscr{E}^{\prime}(K)\right]_{[\theta]} \simeq \mathscr{B}_{p_{\theta}(\cdot)} \cap \mathscr{E}^{\prime}(K)$ (complex interpolation) where $0<\theta<1$ and $\frac{1}{p_{\theta}(x)}=\frac{1-\theta}{p_{0}(x)}+\frac{\theta}{p_{1}(x)}\left(\right.$ we know that $\left[\mathscr{B}_{p_{0}(\cdot)}, \mathscr{B}_{p_{1}(\cdot)}\right]_{[\theta]} \simeq \mathscr{B}_{p_{\theta}(\cdot)}$ (use the definition of $\mathscr{B}_{p(\cdot)}$ and [3, Theorem 7.1.2]); see also [23, pp. 66-78]).

\section{Acknowledgements}

Partially supported by grants MTM2008-04594 and MTM2011-23164 from the Spanish Ministry of Science and Innovation.

\section{References}

[1] D. Cruz-Uribe, D., SFO, A. Fiorenza, J. M. Martell, C. Pérez, C., The boundedness of classical operators on variable $L^{p}$ spaces, Ann. Acad. Sci. Fenn. Math. 31 (2006), 239-264.

[2] L. Diening, Maximal function on Musielak-Orlicz spaces and generalized Lebesgue spaces, Bull. Sci. Math. 129 (2005), 657-700.

[3] L. Diening, P. Harjulehto, P. Hästö, M. Růžička, Lebesgue and Sobolev Spaces with Variable Exponents, Lecture Notes in Mathematics 2017. Springer-Verlag, BerlinHeidelberg (2011).

[4] C. Fefferman, The multiplier problem for the ball, Ann. of Math. (2) 94 (1971), 330-336.

[5] J. García-Cuerva, J. L. Rubio de Francia, Weighted norm inequalities and related topics, North-Holland Mathematical Studies, vol. 116, Amsterdam (1985).

[6] V. Hermanns, Zur Existenz von Rechtsinversen linearer partieller Differentialoperatoren mit konstanten Koeffizienten auf $\mathscr{B}_{p, k}^{\text {loc }}$-Räumen, Dissertation, Wuppertal (2005).

[7] M. Hoffmann, The Banach envelope of Paley-Wiener type spaces, Proc. Amer. Math. Soc. 131 (2002), 543-548.

[8] L. Hormander, The Analysis of Linear Partial Operators II, Grundlehren vol. 257, Springer-Verlag, Berlin-Heidelberg (1983).

[9] H. Jarchow, Locally Convex Spaces, Teubner-Verlag, Stuttgart (1981).

[10] G. Köthe, Topological Vector Spaces I, Springer-Verlag, Berlin-Heidelberg (1969).

[11] O. Kováčik, J. Rákosník, On spaces $L^{p(x)}$ and $W^{p(x)}$, Czechoslovak Math. J. 41 (116) (1991), 592-618.

[12] J. Lindenstrauss, L. Tzafriri, Classical Banach Spaces I, Springer-Verlag, BerlinHeidelberg (1977).

[13] O. Mendez, M. Mitrea, The Banach Envelopes of Besov and Triebel-Lizorkin Spaces and Applications to Partial Differential Equations, J. Fourier Anal. Appl. 6 (2000), 503-531.

[14] B. S. Mitiagin, On idempotent multipliers in symmetric functional spaces, Funkcional Anal. i Prilozen 6 (1972), 81-82.

[15] J. Motos, M. J. Planells, C. F. Talavera, On some iterated weighted spaces, J. Math. Anal. Appl. 338 (2008), 162-174.

[16] J. Motos, M. J. Planells, On sequence space representations of Hörmander-Beurling spaces, J. Math. Anal. Appl. 348 (2008), 395-403.

[17] J. Motos, M. J. Planells, J. Villegas, Some embedding theorems for Hörmander-Beurling spaces, J. Math. Anal. Appl. 364 (2010), 473-482.

[18] J. Motos, M. J. Planells, C. F. Talavera, On variable exponent Lebesgue spaces of entire analytic functions, J. Math. Anal. Appl. 388 (2012), 775-787. 
[19] S. M. Nikol'skij, Approximation of Functions of Several Variables and Imbedding Theorems, Springer-Verlag, Berlin-Heidelberg (1975).

[20] H. J. Schmeisser, H. Triebel, Topics in Fourier Analysis and Function Spaces, John Wiley \& Sons, Chichester (1987).

[21] L. Schwartz, Théorie des distributions, Hermann, Paris (1966).

[22] H. Triebel, Interpolation Theory, Function Spaces, Differential Operators, NorthHolland, Amsterdam (1978).

[23] H. Triebel, Theory of Function Spaces, Birkhäuser, Basel (1983).

[24] D. Vogt, Sequence space representations of spaces of test functions and distributions, In: G. I. Zapata (ed.) Functional analysis, holomorphy and approximation theory, Lecture Notes in Pure and Applied Mathematics, no. 83 (1983), 405-443.

\author{
Joaquín Motos \\ Departamento de Matemática Aplicada \\ Universidad Politécnica de Valencia \\ Camino de Vera, $\mathrm{s} / \mathrm{n}$ \\ 46022 Valencia (Spain) \\ e-mail: jmotos@mat.upv.es \\ María Jesús Planells \\ Departamento de Matemática Aplicada \\ Universidad Politécnica de Valencia \\ Camino de Vera, s/n \\ 46022 Valencia (Spain) \\ e-mail: mjplanells@mat .upv.es \\ César Felix Talavera \\ Departamento de Matemática Aplicada \\ Universidad Politécnica de Valencia \\ Camino de Vera, s/n \\ 46022 Valencia (Spain) \\ e-mail: talavera@mat.upv.es
}

